\title{
Metodologia da Problematização e Aprendizagem Baseada em Problemas na Odontologia: análise bibliométrica dos trabalhos apresentados nas Reuniões da SBPqO
}

\author{
Niebla Bezerra de Melo*; José de Alencar Fernandes Neto*; Maria Helena Chaves de Vasconcelos \\ Catão**; Patrícia Meira Bento** $^{* *}$ \\ * Mestrando(a) em Odontologia do Programa de Pós-Graduação \\ em Odontologia da UEPB \\ ** Professora Doutora do Programa de Pós-Graduação em \\ Odontologia da UEPB \\ Recebido em 23/03/2017. Aprovado em 06/05/2017.
}

\begin{abstract}
RESUMO
Este estudo teve como objetivo investigar a produção científica brasileira sobre Metodologia da Problematização (MP) e Aprendizagem Baseada em Problemas (PBL) na Odontologia. Tratou-se de uma pesquisa transversal, com abordagem quantitativa, a partir de dados secundários. Para tal, realizou-se uma análise bibliométrica dos resumos apresentados nas últimas dez Reuniões da Sociedade Brasileira de Pesquisa Odontológica - SBPqO (2007-2016). A pesquisa dos trabalhos procedeu-se através da seguinte estratégia: localização da sigla "PBL" ou do fragmento "problem" entre os resumos publicados nos anais do evento. Após aplicação dos critérios de inclusão e exclusão, dos 902 resumos encontrados, excluiu-se 890, permanecendo apenas 12 resumos. Desses, $9(75,0 \%)$ foram pesquisas realizadas em universidades públicas, $8(66,7 \%)$ na região Sudeste do Brasil e apenas $1(8,3 \%)$ recebeu apoio financeiro. Apesar da MP e do PBL serem considerados metodologias ativas importantes no processo de ensino-aprendizagem, diante dos resultados encontrados nesse estudo, conclui-se que poucas pesquisas abordando esses métodos na Odontologia foram desenvolvidas nos últimos dez anos no Brasil, de acordo com os trabalhos apresentados nas Reuniões da SBPqO.

Descritores: Aprendizagem Baseada em Problemas. Odontologia. Ensino. Aprendizagem. Pesquisa em Odontologia.
\end{abstract}

\section{INTRODUÇÃO}

Segundo as Diretrizes Curriculares Nacionais para o Ensino de Graduação em Odontologia, o ensino Odontológico deve seguir um projeto pedagógico, centrado no aluno como sujeito e apoiado no professor como facilitador e mediador do processo ensino-aprendizagem, visando à formação integral e adequada do estudante através da articulação entre o ensino, a pesquisa e a extensão ${ }^{1,2}$.

Dessa forma o ensino tradicional na Odontologia tem passado por uma série de questionamentos e mudanças. As principais dificuldades encontradas neste modelo surgem 
devido ao fato da presença de padrões curriculares fragmentados, estruturados em disciplinas básicas e específicas; e apresentação de aulas para grandes grupos, onde o professor é o centro do processo, detentor do conhecimento ${ }^{3-5}$.

Nesse contexto, a prática de metodologias ativas realizadas no processo educacional em cursos da Área da Saúde é uma estratégia que coloca o aluno no centro, estimulando $\mathrm{e}$ ampliando a sua capacidade criativa e gerencial, por meio de processos interativos de conhecimento, análises, estudos, pesquisas e decisões individuais ou coletivas ${ }^{5,6}$.

São muitas as possibilidades de estratégias que focam no aluno como centro do processo de aprendizagem. Dentre elas, destacam-se a Metodologia da Problematização (MP) e a Aprendizagem Baseada em Problemas (Problem Based Learning - PBL) ${ }^{7,8}$.

A MP tem sido cada vez mais utilizada na área da saúde como uma estratégia de ensino, pois permite que os estudantes, sob a tutela do professor, iniciem o processo de construção do conhecimento partindo da realidade observada, de forma mais ampla e profunda, permitindo a sua participação ativa no processo de aprendizagem $^{9-11}$.

A MP é uma estratégia utilizada em situações nas quais os temas estão diretamente relacionados com a vida cotidiana e tem como referência um importante método, o "Arco de Charles Maguerez" ou "Método do Arco" que foi apresentado pela primeira vez, em 1982, por Bordenave e Pereira ${ }^{12,13}$. A metodologia, é desenvolvida a partir de cinco etapas: observação da realidade e definição de um problema, formulação de pontos chave, teorização, hipóteses de solução, e aplicação à realidade ${ }^{14,15}$.

$\mathrm{O}$ uso da problematização como metodologia de ensino é indicado quando os conteúdos ou temas a serem abordados estão relacionados com a vida em sociedade, apresentando os problemas e estimulando a resolução dos mesmos pelos alunos ${ }^{16}$.

No caso do PBL, as situações são preparadas previamente, de acordo com os conteúdos que os alunos precisam dominar. $\mathrm{O}$ método é considerado uma ferramenta de ensino que promove o desenvolvimento do raciocínio clínico integrado, destacando as habilidades cognitivas de diagnóstico e resolução de problemas $^{5,17}$. O PBL foi introduzido pela primeira vez no ensino odontológico na Faculdade de Malmö, na Suécia, em 1990 ${ }^{18-20}$.

O PBL é desenvolvido por meio da formação de pequenos grupos de alunos trabalhando juntos na solução de um problema, sob orientação de um tutor, a partir das seguintes etapas: apresentação de uma situação problema; estudo e análise da situação; formação de hipóteses de trabalho; formação de questões de aprendizagem; compartilhamento e avaliação das habilidades adquiridas pelo aluno durante $\mathrm{o}$ processo $^{5,21,22}$.

Apesar de semelhantes, a MP e o PBL são métodos específicos, com estrutura e procedimentos próprios, não sendo sinônimos em termos de metodologia de ensino ${ }^{14}$. No entanto, ambos contribuem para a formação integral do estudante, partindo de formulações de problemas baseados em situações reais, facilitando a aplicação do conhecimento na prática.

Diante disso, neste estudo teve-se como objetivo investigar a produção científica brasileira sobre o MP e PBL na Odontologia, traçando o perfil dos trabalhos apresentados nas Reuniões da Sociedade Brasileira de Pesquisa Odontológica (SBPqO).

\section{MATERIAL E MÉTODOS}

Este estudo tratou-se de uma pesquisa transversal, com abordagem quantitativa, a partir de dados secundários. 
Realizou-se uma análise dos resumos apresentados nas últimas dez Reuniões da SBPqO (2007-2016) publicados nos anais do evento, disponíveis online em seu site ${ }^{23}$. Escolheu-se os trabalhos das Reuniões da $\mathrm{SBPqO}$, por se tratar do evento científico que mais reúne pesquisas em Odontologia de todo o Brasil. Todas as categorias dos anais foram analisadas e todos os dados utilizados neste estudo são de acesso público.

As etapas descritas a seguir foram realizadas, separadamente, por dois pesquisadores, alunos de pós-graduação, previamente calibrados.

Para pesquisa dos resumos procedeu-se a seguinte estratégia: localização da sigla "PBL" ou do fragmento "problem" entre os resumos dos anais. A ferramenta de busca garantiu que todas as palavras que continham os fragmentos fossem identificadas, mesmo que estivessem separadas por hífen. Dessa forma, todos os trabalhos que continham em seu texto "Aprendizagem Baseada em Problemas", "Ensino Baseado em Problemas", "problematização", "Metodologia da Problematização", "Problematization Methodology", "Problem Based Learning" ou "PBL" puderam ser identificados e posteriormente analisados.

Foram incluídos para análise final todos os trabalhos que atenderam os seguintes critérios de inclusão: resumos que claramente abordaram MP e/ou PBL, originados de pesquisas desenvolvidas por uma instituição de ensino e publicados nos anais de 2007 a 2016, na língua portuguesa ou inglesa. Os resumos que suscitaram dúvidas aos avaliadores durante a fase de triagem foram mantidos para uma avaliação mais detalhada, através de uma reunião de consenso entre os dois examinadores. Permanecendo a dúvida, os mesmos foram encaminhados a um terceiro avaliador (professor expert no assunto).

Nos resumos que obedeceram aos critérios de inclusão foram colhidas as seguintes informações: tipo de instituição (pública ou privada) e estado brasileiro onde a pesquisa foi realizada, recebimento de auxílio financeiro e área em que o resumo foi inserido nos anais. Os dados foram tabulados e avaliados com auxílio do software IBM SPSS Statistics versão 20.0 (IBM Corp., Armonk, NY, USA).

\section{RESULTADOS}

Inicialmente, foram encontrados 902 resumos que continham os fragmentos utilizados. Após análise, foram excluídos 890 estudos, por não obedecerem aos critérios de inclusão da pesquisa, permanecendo, assim, apenas 12 resumos. Nas Reuniões de 2008, 2011, 2012 e 2015, nenhum trabalho envolvendo MP ou PBL foi encontrado (quadro 1).

Os resumos selecionados que envolveram a metodologia da problematização ou PBL, apresentados nas Reuniões da $\mathrm{SBPqO}$, de acordo com ano de publicação dos anais estão dispostos no quadro 2.

Das 12 pesquisas encontradas, $9(75,0 \%)$ foram realizadas em universidades públicas, 8 $(66,7 \%)$ foram desenvolvidas na região Sudeste do Brasil e apenas $1(8,3 \%)$ recebeu auxílio financeiro (tabela 1).

\section{DISCUSSÃO}

A utilização de metodologias ativas ainda é um desafio para os profissionais da educação. Faz-se necessário mudanças de paradigma no ensino e maiores investimento em formação específica apropriada, possibilitando a formação de sujeitos críticos e reflexivos ${ }^{13}$. Além disso, a pesquisa científica de tais estratégias torna-se essencial para o conhecimento e aplicação dos métodos de maneira eficaz.

As ciências da saúde são áreas de estudo relacionadas à vida, saúde e doença dos seres humanos, dessa forma o processo educacional deve acontecer de forma complexa e integrada ${ }^{5}$. Para isso, têm-se estudado as metodologias ativas, que são estratégias de ensino e aprendizagem voltadas para a integralização do conhecimento. 
Quadro 1 - Análise dos resumos publicados, encontrados, excluídos e selecionados de acordo com ano das Reuniões da SBPqO

\begin{tabular}{|c|c|c|c|c|}
\hline Reunião-Ano & $\begin{array}{c}\text { Resumos } \\
\text { publicados }\end{array}$ & $\begin{array}{c}\text { Resumos encontrados } \\
\text { (PBL/Problem) }\end{array}$ & $\begin{array}{c}\text { Resumos } \\
\text { excluídos }\end{array}$ & $\begin{array}{c}\text { Resumos } \\
\text { selecionados }\end{array}$ \\
\hline $24^{\mathrm{a}}-2007$ & 2.588 & 81 & 78 & 3 \\
\hline $25^{\mathrm{a}}-2008$ & 2.373 & 77 & 77 & 0 \\
\hline $26^{\mathrm{a}}-2009$ & 2.648 & 87 & 83 & 4 \\
\hline $27^{\mathrm{a}}-2010$ & 2.962 & 121 & 120 & 1 \\
\hline $28^{\mathrm{a}}-2011$ & 2.873 & 115 & 115 & 0 \\
\hline $29^{\circ}-2012^{*}$ & 16 & 0 & 0 & 2 \\
\hline $30^{\mathrm{a}}-2013$ & 2.804 & 112 & 110 & 1 \\
\hline $31^{\mathrm{a}}-2014$ & 2.752 & 101 & 100 & 0 \\
\hline $32^{\mathrm{a}}-2015$ & 2.926 & 110 & 110 & 1 \\
\hline $33^{\circ}-2016$ & 3.207 & 98 & 97 & 12 \\
\hline Total & 25.149 & 902 & 890 & 0 \\
\hline
\end{tabular}

*A $29^{\mathrm{a}}$ Reunião ocorreu em conjunto com a Reunião da Internacional Association for Dental Research (IADR)

Quadro 2 - Resumos de pesquisas sobre Metodologia da problematização e Aprendizagem Baseada em Problemas apresentados nas Reuniões da SBPqO, de acordo com ano de publicação dos anais

\begin{tabular}{|c|c|}
\hline Reunião - Ano & Resumos selecionados \\
\hline \multirow{3}{*}{$24^{\mathrm{a}}-2007$} & $\begin{array}{l}\text { Novas metodologias de ensino em saúde bucal coletiva: uma avaliação qualitativa sob o } \\
\text { ponto de vista discente }\end{array}$ \\
\hline & $\begin{array}{l}\text { Curso de Aperfeiçoamento em Saúde Bucal Coletiva: da integração corpo-mente à } \\
\text { experiência vivencial do plano de intervenção }\end{array}$ \\
\hline & $\begin{array}{l}\text { Ensino problematizador: a inversão da teoria pela prática na disciplina de Dentística e } \\
\text { conteúdos do ciclo básico do curso de odontologia }\end{array}$ \\
\hline \multirow{4}{*}{$26^{\mathrm{a}}-2009$} & Integração curricular por meio da problematização de casos \\
\hline & Estação Ensino do Coletivo - educação que te quero transformadora \\
\hline & $\begin{array}{l}\text { Experiência do uso de metodologia ativa de ensino aprendizagem no curso de } \\
\text { odontologia da UEM: avaliação discente }\end{array}$ \\
\hline & $\begin{array}{l}\text { Seminário para a sensibilização da importância do acolhimento e enfoque humanizado: } \\
\text { uma disciplina, um novo olhar }\end{array}$ \\
\hline $27^{a}-2010$ & $\begin{array}{l}\text { Inserção da pedagogia problematizadora associada ao ambiente virtual para o } \\
\text { comprometimento e motivação do discente }\end{array}$ \\
\hline \multirow{2}{*}{$29^{\circ}-2013$} & $\begin{array}{l}\text { Radiologia e Materiais dentários: Modelo de Ensino-Aprendizado (Ensino Baseado em } \\
\text { Problemas - PBL) }\end{array}$ \\
\hline & $\begin{array}{l}\text { A Metodologia da Problematização como alternativa de abordagem de ensino } \\
\text { aprendizagem na formação do futuro docente: Arco de Maguerez }\end{array}$ \\
\hline $30^{a}-2014$ & $\begin{array}{l}\text { Metodologias Ativas de Ensino-Aprendizagem: percepções dos acadêmicos de } \\
\text { Odontologia }\end{array}$ \\
\hline $33^{\circ}-2016$ & $\begin{array}{l}\text { Avaliação do método de aprendizagem baseada em problemas aplicado à alunos de } \\
\text { graduação em odontologia, na disciplina de radiologia }\end{array}$ \\
\hline
\end{tabular}


Tabela 1 - Distribuição dos trabalhos $(\mathrm{n}=12)$ que abordam MP e PBL de acordo com a natureza da instituição, regiões, áreas e recebimento de auxílio financeiro no país

\begin{tabular}{lcc}
\hline Variável & n & \% \\
\hline Instituição & & \\
Pública & 9 & 75,0 \\
Privada & 3 & 25,0 \\
Auxílio Financeiro & & \\
Sim & 1 & 8,3 \\
Não & 11 & 91,7 \\
Região & & \\
Centro-Oeste & 2 & 16,7 \\
Nordeste & 1 & 8,3 \\
Norte & 0 & 0 \\
Sudeste & 8 & 66,7 \\
Sul & 1 & 8,3 \\
Áreas* & & \\
Clínica Odontológica & 1 & 8,3 \\
Diagnóstico I e II / Radiologia & 1 & 8,3 \\
Estomatologia / Odontologia Coletiva III & 1 & 8,3 \\
Odontologia Restauradora & 2 & 16,7 \\
Materiais Dentários & 1 & 8,3 \\
Odontologia & 2 & 16,7 \\
Reabilitação Oral & 1 & 8,3 \\
Saúde e Sociedade & 1 & 8,3 \\
Não declarada & 2 & 16,7 \\
\hline *Áreas da Odontologia, segundo anais das reuniões da SBPqO. & & \\
\hline
\end{tabular}

O PBL é efetivo, principalmente no desenvolvimento de habilidades e raciocínio crítico, porém o método tradicional de ensino deve ser utilizado em conjunto. $\mathrm{O}$ conhecimento aprofundado em determinado assunto tem sua importância diminuída caso não seja aplicado para manejar situações reais. Portanto, o pensamento crítico e a habilidade de resolver problemas estão sendo cada vez mais reconhecidos na formação acadêmica ${ }^{24}$.

Essa metodologia se fundamenta como uma prática pedagógica para qualquer área do ensino superior, favorecendo a construção de um conhecimento significativo e mostrando uma visão moderna e multidisciplinar da entidade de ensino, porém, a utilização do PBL deve estar adequada às particularidades da área de conhecimento, ao grupo de docentes e discentes, à instituição e às diretrizes que regem o Ensino Superior do país ${ }^{25}$.

Rêgo e Rodrigues ${ }^{26}$ (2015) avaliaram a aplicação da MP na formação de estudantes de pós-graduação de odontologia e verificaram que o método de ensino-aprendizagem foi válido para a formação de novos professores. Além disso, todos os participantes, relataram que foram suficientemente encorajados a desenvolver um pensamento crítico sobre as situações abordadas através do método, podendo este ser aplicado como um mecanismo complementar na construção do conhecimento dos futuros professores, além de ser uma estratégia para pesquisa e estudo.

Alrahlah $^{27}$ (2016) em uma revisão crítica 
sobre a eficácia do PBL como método de ensino na Odontologia, encontrou evidências de que estudantes que tiveram contato com o PBL durante os cursos de graduação exibem habilidades profissionais superiores em comparação àqueles que foram instruídos apenas pela metodologia tradicional de ensino. Dentre essas habilidades destacam-se o pensamento crítico, a maior capacidade de resolver problemas e de trabalhar em equipe.

Uma revisão sistemática realizada por Bassir et al. ${ }^{7}$ (2014) comparou a eficácia do PBL com métodos de abordagens tradicionais no ensino da Odontologia e concluiu que o PBL não influencia negativamente a aquisição de conhecimento em estudantes de Odontologia e aumenta a capacidade dos alunos na aplicação de seus conhecimentos a situações clínicas.

Esta pesquisa revelou que a maioria dos trabalhos encontrados, que abordavam a metodologia PBL e MP, foram realizados em instituições públicas e na região Sudeste do Brasil. Isso pode ser justificado pelo fato da maior parte $(61 \%)$ dos programas de pósgraduação em Odontologia do país estarem localizados no Sudeste, o que intensifica o número de pesquisas realizadas nessa região ${ }^{28}$.

Apenas uma pesquisa relatou recebimento de apoio financeiro para o seu desenvolvimento. É evidente a importância das agências de fomento à pesquisa para que se possa desenvolver, concluir e publicar os trabalhos científicos $^{29}$.

Uma das condições para aceder aos financiamentos à pesquisa é a demonstração de produtividade, sobretudo em publicações em veículos acadêmicos de melhor reputação. Dentre os principais produtos científicos gerados na Odontologia, estão as publicações de artigos científicos em periódicos reconhecidos, publicação de livros e apresentação de resumos em importantes eventos científicos, como a
Reunião Anual da $\mathrm{SBPqO}$, que é considerada o principal evento científico nacional da Odontologia ${ }^{30}$.

Faz-se necessária a realização de mais estudos sobre metodologias ativas, como a MP e o PBL, buscando entender suas diferenças, benefícios e desafios no ensino da odontologia, afim de melhorar e garantir a eficácia do ensino e aprendizagem ${ }^{5}$.

\section{CONCLUSÃO}

Apesar da MP e do PBL serem considerados metodologias ativas importantes no processo de ensino-aprendizagem, diante dos resultados encontrados nesse estudo conclui-se que poucas pesquisas abordando esses métodos na Odontologia foram desenvolvidas nos últimos dez anos no Brasil, de acordo com os trabalhos apresentados nas Reuniões da SBPqO.

\section{ABSTRACT \\ Problem-solving Methodology and Problem- Based Learning in Dentistry: bibliometric analysis of papers presented at $\mathrm{SBPqO}$ meetings} This study aimed to investigate the Brazilian scientific production on Problematization Methodology (PM) and Problem-Based Learning (PBL) in Dentistry. It was a transversal research, with a quantitative approach, based on secondary data. For that, a bibliometric analysis of the abstracts presented in the last ten Meetings of the Brazilian Society of Dental Research - SBPqO (2007-2016) was carried out. The research performed using the following strategy: location of the abbreviation "PBL" or the fragment "problem" among the abstracts published in the annals of the event. After applying the inclusion and exclusion criteria, 902 abstracts were found, 890 were excluded, remaining only 12 abstracts. Nine $(75.0 \%)$ were researches carried out in public universities, $8(66.7 \%)$ in the Southeast region of Brazil and only $1(8.3 \%)$ received financial support. Although MP and PBL are considered important active methodologies in the teaching-learning process, given the results 
found in this study, it is concluded that few studies addressing these methods in dentistry have been developed in the last ten years in Brazil, according to the SBPqO Meetings.

Descriptors: Problem-Based Learning. Dentistry. Teaching. Learning. Dental Reseach.

\section{REFERÊNCIAS}

1. Brasil. Ministério da Educação. Conselho Nacional de Educação. Câmara de Educação Superior. Resolução CNE/CES, 19 de fevereiro de 2002. Institui as Diretrizes Curriculares Nacionais do Curso de Graduação em Odontologia. Brasília, 2002. (Acesso em 10 mar. 2017). Disponível em: http://portal.mec.gov.br/cne/arquivos/pdf/C ES032002.pdf.

2. Oliveira RG, Dias AL, Ferraz Júnior AM, Porto FR, Hespanhol FL, Silva RHA et al. Problematização como método ativo de ensino-aprendizagem em um Curso de Odontologia. Rev ABENO. 2015;15(2): 74-81.

3. Paula L, Bezerra ACB. A estrutura curricular dos cursos de Odontologia no Brasil. Rev ABENO. 2003;3(1):7-14.

4. Toassi RFC, Stobäus CD, Mosquera JJM, Moysés SJ. Currículo integrado no ensino de Odontologia. Interface Comun Saúde Educ. 2012;16 (41):529-42.

5. Rocha JS, Dias GF, Campanha NH, Baldani MH. O uso da aprendizagem baseada em problemas na Odontologia: uma revisão crítica da literatura. Rev ABENO. 2016;16 (1):25-38.

6. Freire P. Pedagogia da autonomia: saberes necessários à prática docente. São Paulo: $\mathrm{Paz}$ e Terra. 1996.

7. Bassir SH, Sadr-Eshkevari P, Amirikhorheh $S$, Karimbux NY. Problem-based learning in dental education: a systematic review of the literature. J Dent Educ. 2014;78(1):98-109.

8. Queiroz JRC, Oliveira MFJ, Souza DMC, Araújo ALLS, Fuscella MAP. Aprendiza- gem por projeto e inovação tecnológica: união por competências. Rev ABENO. 2016;16(2):2-6.

9. Berbel, NAN. Metodologia da problematização: respostas de lições extraídas da prática. Semin Ciênc Soc Hum. 2014; 35 (2):61-76.

10. Cotta RMM, Silva LS, Lopes LL, Gomes KO, Cotta FM, Lugarinho $\mathrm{R}$, et al. Construção de portfólios coletivos em currículos tradicionais: uma proposta inovadora de ensino aprendizagem. Ciênc Saúde Coletiva. 2012;17(3):787-96.

11. Lopes JL, Freitas MAO, Domingues TAM, Ohl RIB, Barros ALBL. Metodologia da problematização: estratégia de ensino para a aprendizagem do procedimento de terapia. Ciênc Cuid Saúde. 2016;15(1):187-93.

12. Bordenave J. Pereira A. Estratégias de ensino aprendizagem. 4ed., Petrópolis: Vozes, 1982.

13. Prado ML, Velho MB, Espíndola DS, SSH, Backes VMS. Arco de Charles Maguerez: refletindo estratégias de metodologia ativa na formação de profissionais de saúde. Esc Anna Nery. 2012;16(1):172-7.

14. Vieira MNCM, Panúncio-Pinto MP. Metodologia da Problematização (MP). Medicina (Ribeirão Preto). 2015;48(3): 2418.

15. Fujita JALDM, Carmona EV, Shimo AKK, Mecena EHD. Uso da metodologia da problematização com o Arco de Maguerez no ensino sobre brinquedo terapêutico. Rev Port de Educação. 2016;29(1): 229-58.

16. Reul MA, Lima ED, Irineu $\mathrm{KN}$, Lucas RSCC, Costa EMMB, Madruga RCR. Metodologias ativas de ensino aprendizagem na graduação em Odontologia e a contribuição da monitoria - relato de experiência. Rev ABENO. 2016;16(2):6268. 
17. Alves MNT; Marx M; Bezerra MMM; Landim JMM. Metodologias Pedagógicas Ativas na Educação em Saúde. Id on Line Rev Psic. 2017;10(33): 339-46.

18. Fincham AG, Shuler CF. The changing face of dental education: The impact of PBL. J Dent Educ. 2001; 65(5): 406-21.

19. Neufeld VR, Barrows HS. The "McMaster philosophy": an approach to medical education. J Med Educ. 1974; 49(11):104050 .

20. Haden NK, Hendricson WD, Kassebaum DK, Ranney RR, Weinstein G, Anderson EL, Valachovic RW. Curriculum change in dental education, 2003-09. J Dent Educ. 2010;74(5):539-57.

21. Wang Q, Huiping Li \& Weiguo Pang. From PBL tutoring to PBL coaching in undergraduate medical education: an interpretative phenomenological analysis study. Med Educ Online. 2016; 21:31973. (Acesso em 2 mar. 2017). Disponível em: https://www.ncbi.nlm.nih.gov/pmc/articles/ PMC4939402/.

22. Tsigarides J, Wingfield LR, Kulendran M. Does a PBL-based medical curriculum predispose training in specific career paths? A systematic review of the literature. BMC Res Notes.2017;10(24):1-9.

23. SBPqO: Sociedade Brasileira de Pesquisa Odontológica. (Acesso em 1 mar. 2017). Disponível em: http://www.sbpqo.org.br/ resumos/index.asp

24. Leon LB, Onófrio FQ. Aprendizagem Baseada em Problemas na graduação médica - uma revisão da Literatura atual. Rev Bras Educ Med. 2015; 39(4):614-9.
25. Pinto ML, Mistro FZ, Uemura ST. Ensino baseado em problemas como prática pedagógica aplicada a alunos ingressantes no curso de Odontologia. Rev ABENO. 2016;16(3):28-35.

26. Rêgo HMG, Rodrigues JR. Methodology of problematization with the Maguerez's arch: an alternative method for teaching, research and study in dentistry. Braz Dent Sci. 2015; 18(1):34-43.

27. Alrahlah A. How effective the problembased learning (PBL) in dental education. A critical review. Saudi Dent J. 2016;28(4): 155-61.

28. BRASIL. Ministério da Educação. Coordenação de Aperfeiçoamento de Pessoal de Nível Superior. 2016. (Acesso em 10 mar. 2017). Disponível em: http://www.capes.gov.br/images/documento s/Documentos_de_area_2017/18_odon_doc area_2016.pdf.

29. Oliveira Filho RS, Hochman B, Nahas FX, Ferreira LM. Fomento à publicação científica e proteção do conhecimento científico. Acta Cir Bras. 2005;20(2):35-9.

30. Aquino SN, Martelli DRB, Bonan PRF, Laranjeira AL, Martelli Júnior H. Produção científica odontológica e relação com agências de financiamento de pesquisa. Arq Odontol. 2009;45(3):142-6.

Correspondência para:

Maria Helena Chaves de Vasconcelos Catão

e-mail: mhelenact@ zipmail.com.br

Universidade Estadual da Paraíba

Departamento de Odontologia

Rua Baraúnas, 351 - Bodocongó

58429-500, Campina Grande/PB 